\title{
Bell scenarios in which nonlocality and entanglement are inversely related
}

Giuseppe Vallone, Gustavo Lima, Esteban S. Gomez, Gustavo Canas, Jan-Åke Larsson, Paolo Mataloni and Adan Cabello

\section{Linköping University Post Print}

\section{Tweet}

N.B.: When citing this work, cite the original article.

Original Publication:

Giuseppe Vallone, Gustavo Lima, Esteban S. Gomez, Gustavo Canas, Jan-Åke Larsson, Paolo Mataloni and Adan Cabello, Bell scenarios in which nonlocality and entanglement are inversely related, 2014, Physical Review A. Atomic, Molecular, and Optical Physics, (89), 1, 012102.

http://dx.doi.org/10.1103/PhysRevA.89.012102

Copyright: American Physical Society http://www.aps.org/

Postprint available at: Linköping University Electronic Press http://urn.kb.se/resolve?urn=urn:nbn:se:liu:diva-105764 


\title{
Bell scenarios in which nonlocality and entanglement are inversely related
}

\author{
Giuseppe Vallone, ${ }^{1,2}$ Gustavo Lima, ${ }^{3}$ Esteban S. Gómez, ${ }^{3}$ Gustavo Cañas, ${ }^{3}$ Jan-Åke Larsson, ${ }^{4}$ \\ Paolo Mataloni, ${ }^{2,5}$ and Adán Cabello ${ }^{6}$ \\ ${ }^{1}$ Department of Information Engineering, University of Padova, I-35131 Padova, Italy \\ ${ }^{2}$ Dipartimento di Fisica della "Sapienza" Università di Roma, I-00185 Roma, Italy \\ ${ }^{3}$ Center for Optics and Photonics, MSI-Nucleus on Advanced Optics, Departamento de Física, \\ Universidad de Concepción, 160-C Concepción, Chile \\ ${ }^{4}$ Institutionen för Systemteknik, Linköpings Universitet, SE-58183 Linköping, Sweden \\ ${ }^{5}$ Istituto Nazionale di Ottica (INO-CNR), L.go E. Fermi 6, I-50125 Florence, Italy \\ ${ }^{6}$ Departamento de Física Aplicada II, Universidad de Sevilla, E-41012 Sevilla, Spain
}

(Received 12 July 2013; published 3 January 2014)

\begin{abstract}
We show that for two-qubit chained Bell inequalities with an arbitrary number of measurement settings, nonlocality and entanglement are not only different properties but are inversely related. Specifically, we analytically prove that in absence of noise, robustness of nonlocality, defined as the maximum fraction of detection events that can be lost such that the remaining ones still do not admit a local model, and concurrence are inversely related for any chained Bell inequality with an arbitrary number of settings. The closer quantum states are to product states, the harder it is to reproduce quantum correlations with local models. We also show that, in presence of noise, nonlocality and entanglement are simultaneously maximized only when the noise level is equal to the maximum level tolerated by the inequality; in any other case, a more nonlocal state is always obtained by reducing the entanglement. In addition, we observed that robustness of nonlocality and concurrence are also inversely related for the Bell scenarios defined by the tight two-qubit three-setting $I_{3322}$ inequality, and the tight two-qutrit inequality $I_{3}$.
\end{abstract}

DOI: 10.1103/PhysRevA.89.012102

PACS number(s): 03.65.Ud, 03.67.Bg, 42.50.Xa

\section{INTRODUCTION}

Nonlocality and entanglement are two core concepts in quantum information. If $p_{\rho}(a b)$ is the joint probability that Alice obtains $a=1$ and Bob $b=1$ on a system prepared in state $\rho$, nonlocality is the impossibility of expressing $p_{\rho}(a b)$ as $\sum_{\lambda} p_{\rho}(\lambda) p_{\rho}(a, \lambda) p_{\rho}(b, \lambda)$, where $\lambda$ are preestablished classical correlations [1]. Entanglement is the impossibility of expressing a quantum state as a convex combination of separable states. Nonlocality and entanglement are related concepts in the sense that, to have nonlocality, entanglement is needed [2]. The difference between both concepts has been pointed out before. First, it was noticed that there are entangled states which do not violate specific Bell inequalities [3]. Then, in Ref. [4], the statistical strength of Bell tests was studied, showing that stronger tests (for a given family of Bell inequalities) require nonmaximally entangled states. Similarly, it was shown in [5] that nonmaximally entangled states allow for larger violations (or equivalently a stronger resistance to noise) of the $I_{3}$ two-qutrit inequality [6]. In [7] it was demonstrated that, for general bipartite Bell inequalities with $n$ inputs, $n$ outputs, and $n$-dimensional Hilbert spaces, the entropy of entanglement of the state is essentially irrelevant in obtaining large violation. Finally, in [8,9], it is shown that, for certain inequalities, weakly entangled states outperform maximally entangled ones of arbitrary dimension.

One difficulty in reaching a general conclusion about the relationship between nonlocality and entanglement is that of finding a general scenario where incontrovertible measures of nonlocality and entanglement can be compared. Bipartite scenarios have the advantage that any of the many measures of entanglement assign zero entanglement to product states and maximum entanglement to maximally entangled states [10,11]. Nonlocality is a more delicate issue since different restrictions on the number of measurement settings usually lead to different measures of nonlocality. This suggests that to study such relationship, one needs to consider a general scenario in which each party can perform an arbitrary number of local measurements.

The structure of the paper is the following: In Sec. II we define a measure of nonlocality called robustness of nonlocality that will be used through all the paper. In Sec. III we discuss a general bipartite scenario in which both parties have the same number of settings and prove that, no matter the number of settings, robustness of nonlocality and entanglement are inversely related. We then study how noise affects this conclusion. In Sec. IV we numerically explore the second simplest tight bipartite Bell inequality $I_{3322}$ [12], which has three settings per party, each with two outcomes. In Sec. V, we study a tight two-qutrit Bell inequality $I_{3}$ [6]. In all cases considered we observe the same behavior, namely, that entanglement and robustness of nonlocality are inversely related.

\section{ROBUSTNESS OF NONLOCALITY}

For an ensemble of entangled particles in a state $|\psi\rangle$ and a given Bell inequality, we define the robustness of nonlocality $R$ against loss of local information as the maximum fraction of random particles per observer that can be lost such that the remaining ones can violate the Bell inequality. The robustness of nonlocality is related to the minimum detection efficiency $\eta_{\text {crit }}$ required for a loophole-free violation of the Bell inequality [13] as $R \equiv 1-\eta_{\text {crit }}$.

The idea behind this measure of nonlocality is simple: A violation of a Bell inequality with perfect detection efficiency implies that no local model can reproduce the observed joint 
probabilities. If the minimum detection efficiency is $\eta_{\text {crit }}$, this means that no local model exists, even if one locally rejects a fraction $R$ of the events. Therefore, the larger $R$, the harder it is to reproduce the observed results with local models. Therefore, $R$ may be taken as a measure of nonlocality. As a measure of entanglement we will use the concurrence $[10,11]$.

Any bipartite Bell inequality involving $m_{A}$ and $m_{B}$ dichotomic $( \pm 1)$ observables $A_{j}$ and $B_{k}$ on Alice's and Bob's sides, respectively, can be written in the following form:

$$
\langle\mathcal{S}\rangle_{\rho} \leqslant S_{\mathrm{LHV}}
$$

where $\langle\mathcal{S}\rangle_{\rho}$ is the expectation value of $\mathcal{S}$ in the state $\rho$ and

$$
\mathcal{S}=\sum_{j=1}^{m_{A}} \sum_{k=1}^{m_{A}} c_{j k} p\left(a_{j} b_{k}\right)+\sum_{j=1}^{m_{A}} \alpha_{j} p\left(a_{j}\right)+\sum_{k=1}^{m_{B}} \beta_{k} p\left(b_{k}\right) .
$$

In the previous expression, $p\left(a_{j} b_{j}\right)=p\left(A_{j}=1, B_{k}=1\right)$ are the joint probabilities of detecting the +1 eigenvectors $\left|a_{j}\right\rangle$ and $\left|b_{k}\right\rangle$ of the observables $A_{j}$ and $B_{k}$. If the observables have $d_{A}$ and $d_{B}$ outcomes, any Bell inequality can be expressed in a similar way by using only the first $d_{A}-1$ and $d_{B}-1$ outcomes.

Let us now evaluate the effect of detection inefficiency. For inequalities involving only +1 outcomes such as (2), it is customary to assume that no-detection events do not contribute to the inequality (they can be seen as detection on the " -1 " outcome). However, in order to compute the robustness of nonlocality, it is necessary to optimize over all possible strategies for the no-detection events; for instance, whenever Alice does not get a detection, she can choose to always output +1 for observables $A_{1}$ and output -1 for all other observables [14]. It is worth noting that, instead of grouping inconclusive events with one of the outcomes, different strategies can be used. For instance, a further outcome, corresponding to nondetections, can be added to the observables, [15], or one can also choose to treat nondetections as simply "undefined" [16]. However, these strategies will require a modification of the Bell inequality. In the present paper we will study the robustness of nonlocality by assigning one of the observable outcomes to inconclusive events.

Each strategy giving a definite output to each observable is completely equivalent to relabeling the inputs or outputs of a Bell inequality and using the " -1 " outcome in the case of nondetection for any observable. To give an example, the inequality (2) with Alice giving output +1 only for observable $A_{1}$ in the case of no detection is equivalent to replacing $p\left(a_{1} b_{k}\right) \rightarrow$ $p\left(b_{k}\right)-p\left(a_{1} b_{k}\right)$ and $p\left(a_{1}\right) \rightarrow 1-p\left(a_{1}\right)$ and using the -1 outcome in the case of a no-detection event for any observable.

It can also be noted that from the experimental viewpoint, assigning -1 outcomes for nondetection gives a simple way to handle these events. This is because with this assignment, nodetection events do not contribute to the inequality, so that there is no need to distinguish whether there was a pair produced but no detection, or if there was no pair produced. Distinguishing these are sometimes nontrivial, for example in a continuously pumped experiment, but this is not needed with the suggested assignment.

Thus, since any no-detection strategy is equivalent to rewrite the Bell inequality, the robustness of nonlocality $R$ can be evaluated by optimizing over all possible ways of rewriting the inequality and using the -1 outcome in the case of nondetection (in the case of observable with $d$ outcomes, the last outcome is typically used in the case of nondetection). In order to violate a Bell inequality written as (2), in the case of detection efficiencies $\eta_{A}$ and $\eta_{B}$, the following relation must hold:

$$
\begin{aligned}
& \eta_{A} \eta_{B} \sum_{j=1}^{m_{A}} \sum_{k=1}^{m_{A}} c_{j k} p\left(a_{j} b_{k}\right)+\eta_{A} \sum_{j=1}^{m_{A}} \alpha_{j} p\left(a_{j}\right) \\
& \quad+\eta_{B} \sum_{k=1}^{m_{B}} \beta_{k} p\left(b_{k}\right)>S_{\mathrm{LHV}} .
\end{aligned}
$$

Eberhard first showed that states with lower entanglement allow a violation of the Clauser-Horne-Simony-Holt (CHSH) inequality [17] with lower required detection efficiency [18] with respect to maximally entangled states. Low entangled states tolerate smaller efficiencies when one of the two particles is always detected [19,20]. The same occurs in the $n$-site Clauser-Horne inequality [21]. In [22], it was noticed that nonmaximally entangled states of two qudits can lower the required detection efficiency with respect to maximally entangled states. Recently, it was shown that states with low entanglement can be also useful for Einstein-Podolsky-Rosen (EPR) steering with low detection inefficiencies [23,24].

In the following sections we will demonstrate that states with low entanglement can tolerate lower detection inefficiency for the violation of different Bell inequalities. In particular, we will show that the robustness of non locality $R$ and the entanglement are inversely correlated for the studied inequalities.

\section{ROBUSTNESS OF NONLOCALITY VS CONCURRENCE FOR CHAINED BELL INEQUALITIES}

Pearle [13] and Braunstein and Caves (BC) [25,26] introduced a generalization of the CHSH [17] and Clauser-Horne (CH) [27] Bell inequalities, known as chained Bell inequalities, in which Alice and Bob choose among $M \geqslant 2$ settings. Chained Bell inequalities have some interesting applications: The case $M=3$ fixes a loophole that occurs in some experiments based on the $\mathrm{CHSH}$ inequality [28]. Besides, it reduces the number of trials needed to rule out local hidden variable theories [29], and improves the security of some quantum key distribution protocols [30]. In the case in which $M$ tends to infinity, the inequality allows one to discard nonlocal hidden variable theories with a nonzero local fraction [31]. Chained Bell inequalities have been experimentally tested using pairs of photons, with $M=3$ [32], 4 [33], and 21 [34]. It was recently shown than they can be used for randomness expansion [35].

The version of the chained Bell inequalities introduced in [33], which is symmetric under the permutation of Alice and Bob, reads [by using the notation of (2)]

$$
\left\langle S_{M}\right\rangle_{\rho} \leqslant 0
$$

where

$$
\begin{aligned}
S_{M}= & p\left(a_{M} b_{M}\right)+\sum_{k=2}^{M}\left[p\left(a_{k} b_{k-1}\right)+p\left(a_{k-1} b_{k}\right)\right] \\
& -p\left(a_{1} b_{1}\right)-\sum_{k=2}^{M}\left[p\left(a_{k}\right)+p\left(b_{k}\right)\right] .
\end{aligned}
$$


The minimum detection efficiency required for a loophole-free violation of chained Bell inequalities for any $M \geqslant 2$ using maximally entangled states has been obtained in [36]. The fact that the maximum quantum violation of chained Bell inequalities is always achieved with maximally entangled states [37] might suggest that the minimum detection efficiency occurs for maximally entangled states, but no proof exists of whether the detection efficiency for the chained Bell inequalities can indeed be reduced when one considers more general classes of entangled states. Indeed, for case $M=2$, corresponding to the $\mathrm{CH}$ inequality (that is equivalent the $\mathrm{CHSH}$ ), the minimum detection efficiency occurs for almost product states [18,21].

In the following we will show that, in absence of noise (e.g., considering pure states), the states with higher robustness of nonlocality (or the minimum detection efficiency) for any chained Bell inequality written in the form of (5) are almost product states for which the robustness of nonlocality tends to

$$
R_{M}=\frac{1}{2 M-1} .
$$

The important point here is that this value is larger than the maximum value of $R_{M}$ for maximally entangled states (MES) [36], namely,

$$
R_{M}^{\mathrm{MES}}=\frac{M \cos \left(\frac{\pi}{2 M}\right)-M+1}{M \cos \left(\frac{\pi}{2 M}\right)+M-1} .
$$

Moreover, for Bell inequalities of the form (4) with fixed $M$, we will show that the value in (6) is the maximum achievable robustness of nonlocality for any quantum state. This shows that, for all chained Bell inequalities, entanglement and nonlocality of pure states are inversely related.

Theorem. The maximum of the robustness of nonlocality of inequality (4) is $R_{M}=\frac{1}{2 M-1}$ and can be obtained by almost product state.

Proof. Assuming the same detection efficiency for every party and setting, i.e., $\eta_{A}=\eta_{B}=\eta$, the value of $S_{M}$ becomes

$$
\eta^{2}\left(S_{M}\right)_{\rho}-\eta(1-\eta) \sum_{k=2}^{M}\left[p_{\rho}\left(a_{k}\right)+p_{\rho}\left(b_{k}\right)\right]
$$

where $p_{\rho}\left(a_{k}\right)$ is the expectation value of $p\left(a_{k}\right)$ in the state $\rho$. Therefore, inequality (4) is violated when $\eta>\eta_{\text {crit }}^{(M)}$, with

$$
\eta_{\text {crit }}^{(M)}=\frac{\sum_{k=2}^{M}\left[p_{\rho}\left(a_{k}\right)+p_{\rho}\left(b_{k}\right)\right]}{\left(S_{M}\right)_{\rho}+\sum_{k=2}^{M}\left[p_{\rho}\left(a_{k}\right)+p_{\rho}\left(b_{k}\right)\right]} .
$$

Since $p_{\rho}\left(a_{1} b_{1}\right) \geqslant 0$, it is easy to show that $\left(S_{M}\right)_{\rho}+$ $\sum_{k=2}^{M}\left[p_{\rho}\left(a_{k}\right)+p_{\rho}\left(b_{k}\right)\right] \leqslant p_{\rho}\left(a_{M} b_{M}\right)+\sum_{k=2}^{M}\left[p_{\rho}\left(a_{k} b_{k-1}\right)+\right.$ $\left.p_{\rho}\left(a_{k-1} b_{k}\right)\right]$. Then,

$$
\eta_{\text {crit }}^{(M)} \geqslant \frac{\sum_{k=2}^{M}\left[p_{\rho}\left(a_{k}\right)+p_{\rho}\left(b_{k}\right)\right]}{p_{\rho}\left(a_{M} b_{M}\right)+\sum_{k=2}^{M}\left[p_{\rho}\left(a_{k} b_{k-1}\right)+p_{\rho}\left(a_{k-1} b_{k}\right)\right]} .
$$

Clearly,

$$
0 \leqslant p_{\rho}\left(a_{j} b_{k}\right) \leqslant \min \left[p_{\rho}\left(a_{j}\right), p_{\rho}\left(b_{k}\right)\right],
$$

and the lowest possible bound of the right-hand side of (10) is obtained when $p_{\psi}\left(a_{j} b_{k}\right)=p_{\psi}\left(a_{j}\right)=p_{\psi}\left(b_{k}\right)$ for $j$ and $k$ not both equal to 1 . We obtain

$$
\eta_{\text {crit }}^{(M)} \geqslant \frac{(2 M-2) p_{\psi}\left(a_{1}\right)}{(2 M-1) p_{\psi}\left(a_{1}\right)}=\frac{2 M-2}{2 M-1},
$$

which cannot be achieved exactly, but arbitrarily close by the following procedure: Any generic two-qubit pure states $\rho=$ $|\psi\rangle\langle\psi|$, can be written (in a suitable basis) as

$$
|\psi\rangle=\sin \frac{\gamma}{2}|00\rangle-\cos \frac{\gamma}{2}|11\rangle,
$$

with $0 \leqslant \gamma \leqslant \pi / 2$. Let us consider the following eigenstates:

$$
\begin{aligned}
& \left|a_{1}\right\rangle=\left|b_{1}\right\rangle=\cos \frac{\theta}{2}|0\rangle+\sin \frac{\theta}{2}|1\rangle, \\
& \left|a_{k}\right\rangle=\left|b_{k}\right\rangle=|0\rangle, \text { with } \quad k=2, \ldots, M,
\end{aligned}
$$

and choose $\theta$ such that $\tan ^{2} \frac{\theta}{2}=\tan \frac{\gamma}{2}$. Then, $p_{\rho}\left(a_{1} b_{1}\right)=0$ and the critical efficiency becomes

$$
\eta_{\mathrm{crit}}^{(M)}=\frac{2 M-2}{2 M-3+\frac{2}{1+\tan \gamma / 2}},
$$

which, when $\gamma$ tends to zero (i.e., when the state tends to a product state), tends to

$$
\eta_{\text {crit }}^{(M)} \stackrel{\gamma \rightarrow 0}{\longrightarrow} \frac{2 M-2}{2 M-1} \Rightarrow R_{M} \stackrel{\gamma \rightarrow 0}{\longrightarrow} \frac{1}{2 M-1},
$$

concluding our proof.

We have numerically obtained, by using the method of conjugate gradient, $R_{M}$ as a function $C$ of the pure state used to violate the inequality and compared it with the corresponding maximal achievable violation of the Bell inequality $S_{M}$. Moreover, through exhaustive numerical searches, we have obtained that the form (5) gives the maximum $R$ for any given state (in the specific case of a maximally entangled state this is analytically demonstrated in the Appendix). Note that, for nonmaximally entangled states such as (13), the concurrence is given by $C=\sin \gamma$. The results for $M=2,3,4$ are shown in Fig. 1. We observe that larger violations of $S_{M}$ correspond to lower values of $R_{M}$. From Fig. 1 one can clearly see see that nonlocality (measured by $R$ ) and entanglement (measured by $C$ ) are inversely related: Larger concurrence, allowing larger violation of the inequality, implies lower $R$.

\section{A. Adding noise}

How does noise affect this conclusion? In the presence of white noise, the state becomes $\rho=(1-q)|\psi\rangle\langle\psi|+\frac{q}{4} \mathbb{1}$ and the threshold detection for the chained Bell inequalities efficiency is changed to

$$
\eta_{\text {crit }}^{(M)}=\frac{\sum_{k=2}^{M}\left[p_{\rho}\left(a_{k}\right)+p_{\rho}\left(b_{k}\right)\right]+\frac{q}{1-q}(M-1)}{\left(S_{M}\right)_{\rho}+\sum_{k=2}^{M}\left[p_{\rho}\left(a_{k}\right)+p_{\rho}\left(b_{k}\right)\right]+\frac{q}{2(1-q)}(M-1)} .
$$

In Fig. 2 we show, for three different values of noise ( $q=0.01$, $q=0.05$, and $q=0.1$ ), the dependence of $R_{2}$ and $R_{3}$ and the maximum values of $S_{2}$ and $S_{3}$ with the degree of entanglement of the initial pure state. We observe that, when the noise is different from 0 , the best quantum state giving the lowest threshold is not an almost separable state, but a nonmaximally 


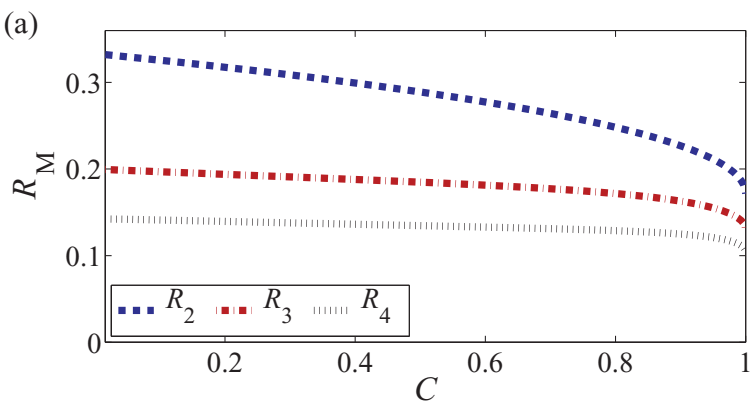

(b)

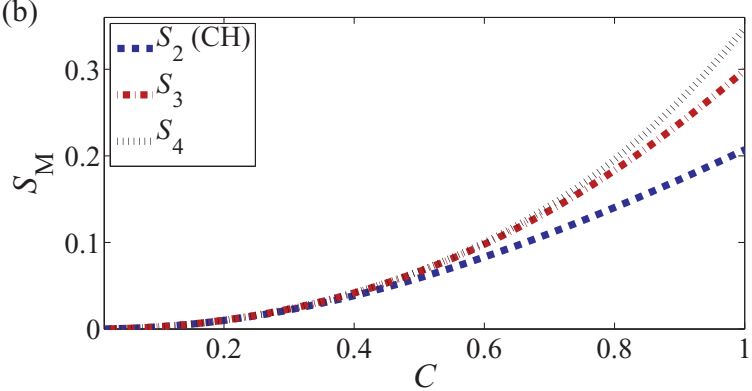

FIG. 1. (Color online) (a) Robustness of nonlocality $R$ as a function of the concurrence $C$. (b) Maximum values of $\left(S_{M}\right)_{\rho}$ violating the chained Bell inequality as a function of $C$.

entangled state depending on $q$. However, the lower the noise $q$, the smaller the entanglement required to obtain the optimal threshold.

Furthermore, in Fig. 2(b) we observe that, the lower $M$ is, the more resistant to noise is the violation of the Bell inequality. In fact, it is possible to calculate the maximum tolerated noise to violate the chained Bell inequalities. Given

(a)

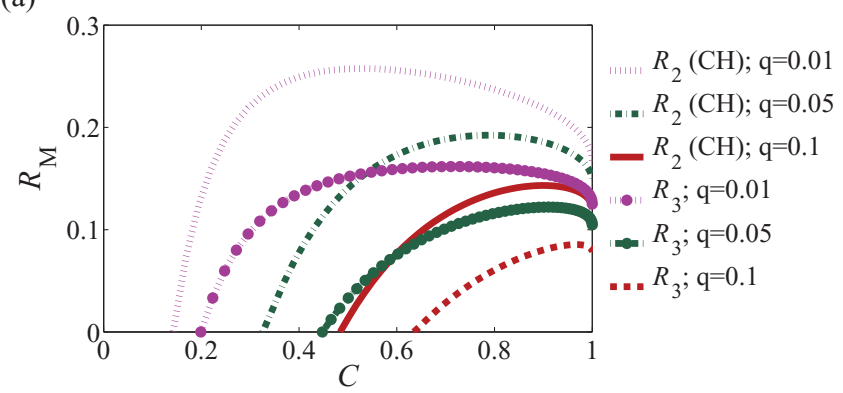

(b)

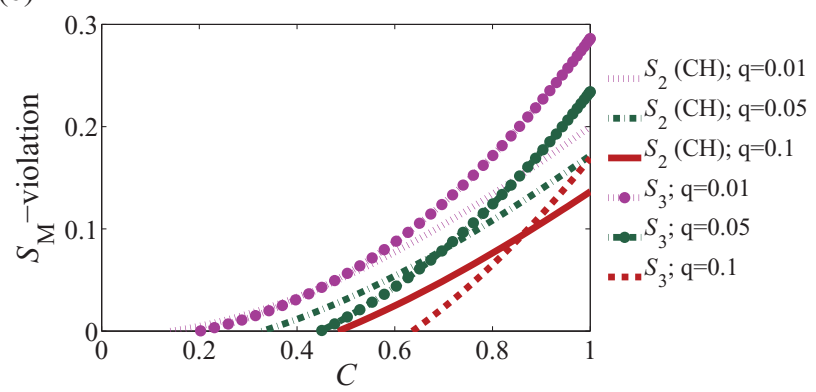

FIG. 2. (Color online) (a) Values of $R_{M}$ and (b) maximum violation of the chained Bell inequality for different number of settings and different degree of noise $(q)$ as a function of the concurrence.

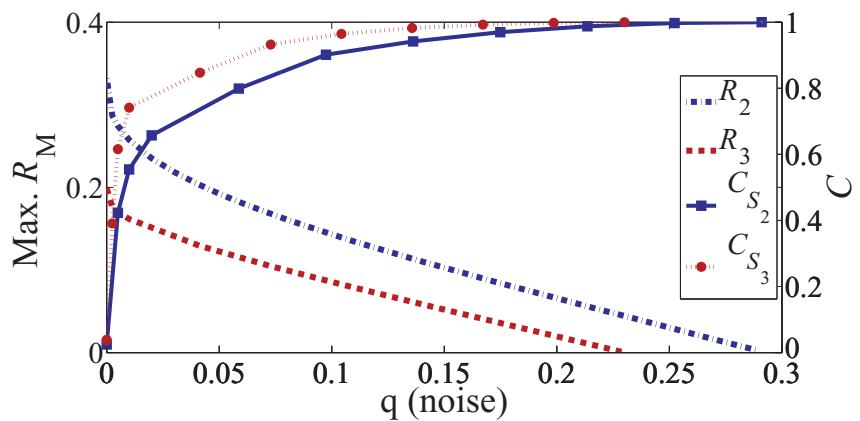

FIG. 3. (Color online) Maximal value of $R_{M}$ in the presence of noise for the $S_{2}$ and $S_{3}$ Bell inequalities. The value of $C$ giving the best $R$ is shown for each $q$.

$\gamma$ and the maximal violation of $S_{M}$ defined as $s_{M}^{\max }(\gamma)$, the maximum tolerated noise is $q_{\max }=\frac{2 s_{M}^{\max }(\gamma)}{2 s_{M}^{\max }(\gamma)+M-1}$.

Using the method of conjugate gradient to minimize Eq. (17), it is also possible to obtain the threshold and the required entanglement for any value of the noise $q$. The results are shown in Fig. 3. We observe that, for chained Bell inequalities, nonlocality and entanglement are simultaneously maximized only in the case of extreme noise, namely the maximum noise level tolerated by the inequality. A better threshold detection efficiency is obtained by lowering the noise and suitably decreasing the entanglement. From this we conclude that nonlocality and entanglement are synonymous only for extremely noisy scenarios.

\section{ROBUSTNESS OF NONLOCALITY VS CONCURRENCE FOR I3322 BELL INEQUALITY}

After the results presented in the previous section, a natural question is whether or not the same behavior occurs for other bipartite Bell inequalities. In this section we present the results for the second simplest tight bipartite Bell inequality, namely, $I_{3322}[12,38,39]$, involving three dichotomic measurements on both $A$ and $B$ sides (the simplest tight bipartite Bell inequality is the CHSH inequality or $S_{2}$, studied in the previous section).

The $I_{3322}$ inequality may be written as

$$
\left\langle I_{3322}\right\rangle_{\rho} \leqslant 0
$$

where $I_{3322}$ was defined in [12] as $I_{3322}=p\left(a_{1} b_{1}\right)+$ $p\left(a_{1} b_{2}\right)+p\left(a_{1} b_{3}\right)+p\left(a_{2} b_{1}\right)+p\left(a_{2} b_{2}\right)+p\left(a_{3} b_{1}\right)-$ $p\left(a_{2} b_{3}\right)-p\left(a_{3} b_{2}\right)-2 p\left(a_{1}\right)-p\left(a_{2}\right)-p\left(b_{1}\right)$. However, this form will not lead to the best $R$. We have numerically checked that the forms giving the best $R$ are the following:

$$
\begin{aligned}
I_{3322}^{(1)}= & p\left(a_{1} b_{1}\right)-p\left(a_{1} b_{2}\right)+p\left(a_{1} b_{3}\right)+p\left(a_{2} b_{1}\right) \\
& -p\left(a_{2} b_{2}\right)+p\left(a_{3} b_{1}\right)-p\left(a_{2} b_{3}\right)+p\left(a_{3} b_{2}\right) \\
& -p\left(a_{1}\right)-p\left(a_{3}\right)-p\left(b_{1}\right),
\end{aligned}
$$

that can be obtained from $I_{3322}$ by replacing $p\left(a_{i} b_{2}\right) \rightarrow$ $p\left(a_{i}\right)-p\left(a_{i} b_{2}\right)$ and

$$
\begin{aligned}
I_{3322}^{(2)}= & -p\left(a_{1} b_{1}\right)-p\left(a_{1} b_{2}\right)+p\left(a_{1} b_{3}\right)-p\left(a_{2} b_{1}\right) \\
& -p\left(a_{2} b_{2}\right)-p\left(a_{3} b_{1}\right)-p\left(a_{2} b_{3}\right)+p\left(a_{3} b_{2}\right) \\
& +p\left(a_{2}\right)+p\left(b_{1}\right)-1,
\end{aligned}
$$


(a)

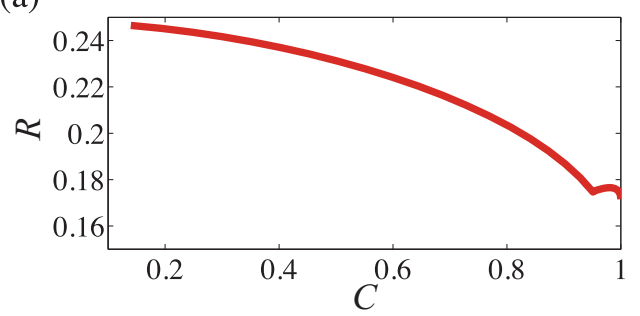

(b)

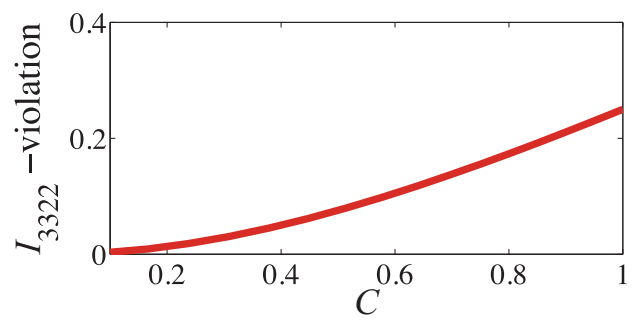

FIG. 4. (Color online) (a) Robustness of nonlocality $R$ as a function of the concurrence $C$ and (b) maximum violation of the $I_{3322}$ inequality as a function of $C$.

obtained from $I_{3322}^{(1)}$ by $p\left(a_{i} b_{1}\right) \rightarrow p\left(a_{i}\right)-p\left(a_{i} b_{1}\right)$ and $p\left(b_{1}\right) \rightarrow 1-p\left(b_{1}\right)$.

Figure 4 shows the $R$ and the violation of the $I_{3322}$ inequality as a function of the degree of entanglement measured by $C$. We observe that, in the absence of noise, almost product states are again those that require lower detection efficiencies. For $C \geqslant 0.9507$ the optimal $R$ is obtained with $I_{3322}^{(2)}$, while for $C<$ 0.9507 the optimal $R$ is obtained with $I_{3322}^{(1)}$. The optimality of $I_{3322}^{(2)}$ can be analytically shown for maximally entangled states (see the Appendix).

The minimum required efficiency with maximally entangled states is 0.8284 as reported in Ref. [14]. Indeed, this value can be obtained analytically. For two-qubit systems, the maximum violation of $I_{3322}$ is $1 / 4$, and can be achieved with a maximally entangled state, as was previously shown in [12]. Given a maximally entangled state $\rho^{\prime}$ that maximally violates $I_{3322}^{(2)}$ and symmetric efficiencies $\eta$, to violate the inequality it is necessary that

$$
\begin{aligned}
& \eta^{2}\left\langle I_{3322}\right\rangle_{\rho^{\prime}}+\eta(1-\eta)\left[p_{\rho^{\prime}}\left(a_{2}\right)-1\right] \\
& \quad+\eta(1-\eta)\left[p_{\rho^{\prime}}\left(b_{1}\right)-1\right]-(1-\eta)^{2}>0 .
\end{aligned}
$$

Remembering that for a maximally entangled state (MES) $p_{\rho^{\prime}}\left(a_{i}\right)=\frac{1}{2}$, we obtain

$$
\eta>2(\sqrt{2}-1) \simeq 0.828 \quad \Rightarrow \quad R^{\mathrm{MES}} \simeq 0.172 .
$$

The maximal robustness of nonlocality $R=\frac{1}{4}$ can be achieved for almost product states. If we consider the $I_{3322}^{(1)}$ form of the inequality, the critical efficiency can be written as $\eta_{c}=\frac{p_{\rho}\left(a_{1}\right)+p_{\rho}\left(a_{3}\right)+p_{\rho}\left(b_{1}\right)}{\left\langle I_{3322}^{(1)}\right\rangle+p_{\rho}\left(a_{1}\right)+p_{\rho}\left(a_{3}\right)+p_{\rho}\left(b_{1}\right)}$. Let us choose $\left|a_{1}\right\rangle=$ $\left|b_{1}\right\rangle=\left|a_{3}\right\rangle=|0\rangle,\left|a_{2}\right\rangle=\cos \frac{\theta}{2}|0\rangle+\sin \frac{\theta}{2}|1\rangle,\left|b_{2}\right\rangle=\left|b_{3}\right\rangle=$ $\sin \frac{\theta}{2}|0\rangle+\cos \frac{\theta}{2}|1\rangle,|\psi\rangle=\sin \frac{\gamma}{2}|00\rangle+\cos \frac{\gamma}{2}|11\rangle$. By using $\theta$ such that $\cos \theta=\frac{1+\cos \gamma}{2(1+\sin \gamma)}$ we obtain

$$
\eta_{\text {crit }}=\frac{12(1+\sin \gamma)}{13+3 \cos \gamma+12 \sin \gamma},
$$

which, when $\gamma$ tends to zero tends to

$$
\eta_{\text {crit }}^{(M)} \stackrel{\gamma \rightarrow 0}{\longrightarrow} \frac{3}{4} \Rightarrow R \stackrel{\gamma \rightarrow 0}{\longrightarrow} \frac{1}{4} .
$$

We observe that the maximum $R$ for the $I_{3322}$ is greater than the one for the $S_{3}$ inequality, which has the same number of local settings.

\section{ROBUSTNESS OF NONLOCALITY VS CONCURRENCE FOR THE $I_{3}$ TWO-QUTRIT INEQUALITY}

For the two-qubit Bell inequalities discussed above we have observed that nonlocality and entanglement are inversely related. Here we show that this is also true for other bipartite scenarios. For this purpose we repeat our analysis but now for a tight bipartite inequality maximally violated by two-qutrit states, the $I_{3}$ inequality [6].

The inequality is given by $\widetilde{I}_{3}=P\left(A_{1}=B_{1}\right)+P\left(B_{1}=\right.$ $\left.A_{2}+1\right)+P\left(A_{2}=B_{2}\right)+P\left(B_{2}=A_{1}\right)-P\left(A_{1}=B_{1}-1\right)-$ $P\left(B_{1}=A_{2}-P\left(A_{2}=B_{2}-1\right)-P\left(B_{2}=A_{1}-1\right) \leqslant 2\right.$, where $P\left(A_{m}=B_{n}+k\right)=\sum_{j=1}^{3} P\left(a_{m}^{j} b_{n}^{j+k \bmod 3}\right)$. Here, $n$ and $m(n, m=1,2)$ denote the settings that the parties may choose for the local measurements, and the index $j$ denotes each measurement outcome $(j=1,2,3)$. The inequality can be rewritten in the form of (2) as $\left\langle I_{3}\right\rangle_{\rho} \leqslant 0$, with

$$
\begin{aligned}
I_{3}= & p\left(a_{1} b_{1}\right)+p\left(a_{1} b_{2}\right)+p\left(a_{2} b_{1}\right)-p\left(a_{2} b_{2}\right)+p\left(\bar{a}_{1} \bar{b}_{1}\right) \\
& +p\left(\bar{a}_{1} \bar{b}_{2}\right)+p\left(\bar{a}_{2} \bar{b}_{1}\right)-p\left(\bar{a}_{2} \bar{b}_{2}\right)+p\left(a_{1} \bar{b}_{1}\right)+p\left(\bar{a}_{1} b_{2}\right) \\
& +p\left(\bar{a}_{2} b_{1}\right)-p\left(\bar{a}_{2} b_{2}\right)-p\left(a_{1}\right)-p\left(\bar{a}_{1}\right)-p\left(b_{1}\right)-p\left(\bar{b}_{1}\right) .
\end{aligned}
$$

In the previous expression $a_{j}$ and $b_{j}$ denote the 1 eigenstates of the observables $A_{j}$ and $B_{k}$, while $\bar{a}_{j}$ and $\bar{b}_{j}$ denote the 2 eigenstates. Note that no probability containing the 3 eigenstate is present. Moreover, $\widetilde{I}_{3}=3 I_{3}+2$.

In Fig. 5 we show the maximal achievable violation of $I_{3}$ in function of the concurrence $C$ while considering the initial two-qutrit state given by $|\psi\rangle=\cos \frac{\theta_{1}}{2} \cos \frac{\theta_{2}}{2}|11\rangle+$ $\cos \frac{\theta_{1}}{2} \sin \frac{\theta_{2}}{2}|22\rangle+\sin \frac{\theta_{1}}{2}|33\rangle$, where $\theta_{k} \in[0, \pi]$. The maximally entangled state is obtained when $\theta_{2}=\pi / 2$ and $\theta_{1}=$

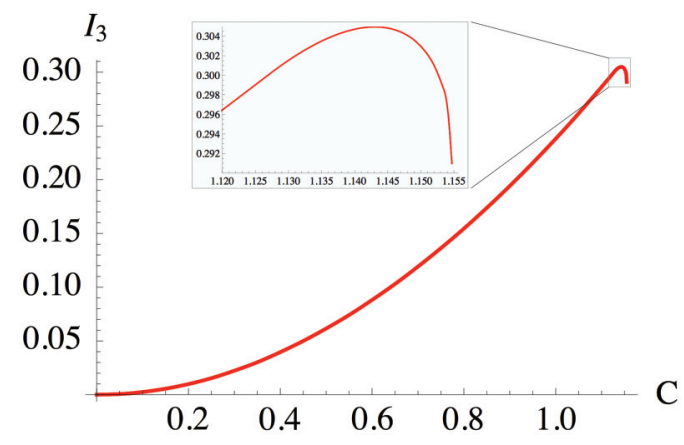

FIG. 5. (Color online) Maximum violation of the $I_{3}$ qutrit inequality as a function of the concurrence $C$. 


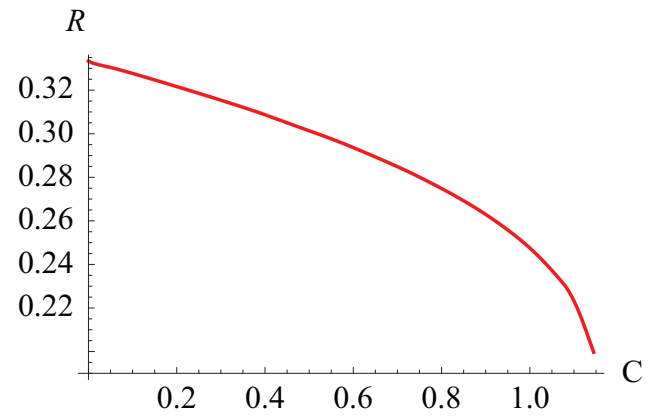

FIG. 6. (Color online) $R$ as a function of the concurrence $C$ for the $I_{3}$ qutrit inequality.

$2 \arcsin \frac{1}{\sqrt{3}}$. The concurrence for the two-qutrit state described above is given by $C=\sqrt{\sin ^{2} \theta_{1}+\sin ^{2} \theta_{2} \cos ^{4} \frac{\theta_{1}}{2}}$ and ranges from 0 to $\frac{2}{\sqrt{3}}$ [11]. As was first observed in [5], the maximal violation of $I_{3}$ is obtained with partially entangled states. The maximal value achievable with MES is $I_{3}^{\mathrm{MES}}=\frac{2}{27}(4 \sqrt{3}-$ $3) \simeq 0.291$ and one can clearly see that it does not correspond to the maximum of $I_{3}$.

We then numerically optimized the robustness of nonlocality $R$ in a function of $C$, by obtaining the results shown in Fig. 6: the form of $I_{3}$ given in (25) is the optimal form for maximizing the $R$ parameter. The optimality can be analytically shown for maximally entangled states (see the Appendix). Also in this case $R$ and entanglement are negatively correlated.

The maximal $R$ is $\frac{1}{3}$ and can be obtained for almost product states, as in the previous inequalities. Let us consider the following entangled state:

$$
|\psi\rangle=\cos \frac{\gamma}{2}|11\rangle+\sin \frac{\gamma}{2}|33\rangle,
$$

and the following measurements: $\left|a_{1}\right\rangle=\left|b_{1}\right\rangle=|3\rangle$, $\left|\bar{a}_{1}\right\rangle=\left|\bar{b}_{1}\right\rangle=|2\rangle, \quad\left|a_{2}\right\rangle=\cos \frac{\theta}{2}|1\rangle+\sin \frac{\theta}{2}|3\rangle, \quad\left|b_{2}\right\rangle=$ $\sin \frac{\theta}{2}|1\rangle+\cos \frac{\theta}{2}|3\rangle, \quad\left|\bar{a}_{2}\right\rangle=\left|\bar{b}_{2}\right\rangle=|2\rangle$. In the form (25) the threshold efficiency becomes (nondetection events correspond to 3 eigenvalues and thus does not contribute to the inequality)

$$
\eta_{c}=\frac{p_{\rho}\left(a_{1}\right)+p_{\rho}\left(\bar{a}_{1}\right)+p_{\rho}\left(b_{1}\right)+p_{\rho}\left(\bar{b}_{1}\right)}{\left\langle\widetilde{I}_{3}\right\rangle_{\rho}+p_{\rho}\left(a_{1}\right)+p_{\rho}\left(\bar{a}_{1}\right)+p_{\rho}\left(b_{1}\right)+p_{\rho}\left(\bar{b}_{1}\right)} .
$$

With the above measurement the critical efficiency becomes

$$
\eta_{c}=\frac{4(1-\cos \gamma)}{3-\cos \theta(2+\cos \theta)-4 \cos \gamma+\sin ^{2} \theta \sin \gamma} .
$$

If we choose $\cos \theta=-\frac{1}{\sin \gamma+1}$ and let $\gamma$ go to zero we get

$$
\eta_{c} \stackrel{\gamma \rightarrow 0}{\longrightarrow} \frac{2}{3} \Rightarrow R \stackrel{\gamma \rightarrow 0}{\longrightarrow} \frac{1}{3} .
$$

For maximally entangled states, for which $p\left(a_{j}\right)=p\left(\bar{a}_{j}\right)=$ $p\left(b_{j}\right)=p\left(\bar{b}_{j}\right)=\frac{1}{3}$, the $R$ is given by

$$
R^{\mathrm{MES}}=\frac{I_{3}^{\mathrm{MES}}}{I_{3}^{\mathrm{MES}}+4 / 3}=\frac{4 \sqrt{3}-3}{4 \sqrt{3}+15} \simeq 0.1791 .
$$

\section{CONCLUSIONS}

We would argue that robustness of nonlocality $R$ is a good measure of nonlocality, since it marks the border where local hidden variable descriptions become possible: The larger robustness of nonlocality is, the harder it is to express the joint probabilities with local models.

We have shown that, for the two-party $M$-setting chained Bell scenario (for any $M \geqslant 2$ finite), for a tight two-qubit Bell inequality $I_{3322}$, and a tight two-qutrit Bell inequality $I_{3}$, robustness of nonlocality and concurrence are, in the absence of noise, inversely related.

The main result of this paper is the observation that, for many distinct types of Bell scenarios, larger nonlocality requires smaller entanglement; in the absence of noise, almost product states are the most nonlocal ones. We analytically showed that the maximal $R$ can be achieved with almost product state. The maximal values of $R$ (related to the minimum required detection efficiency as $\eta_{c}=1-R$ ) are given by $R=\frac{1}{2 M-1}, R=\frac{1}{4}$, and $R=\frac{1}{3}$ for the $S_{M}$ chained Bell inequality, the $I_{3322}$ inequality and the $I_{3}$ inequality respectively.

When noise is present, the most nonlocal states acquire some amount of entanglement; however, the smaller the noise is, the lower their entanglement becomes.

Some questions naturally arise: Are the nonlocality and entanglement inversely related in any Bell inequality involving $m_{A}, m_{B}$ observables with $d_{A}$ and $d_{B}$ outcomes? If yes, is there some physical mechanism for such counterintuitive behavior? These questions require further research.

\section{ACKNOWLEDGMENTS}

G.L., E.S.G., and G.C. were supported by the CONICYT, AGCI, FONDECYT 1120067, MilenioP10-030-F, and PIACONICYT PFB0824. G.V. was supported by the StrategicResearch-Project QUINTET of the Department of Information Engineering, University of Padova and the Strategic-ResearchProject QUANTUMFUTURE of the University of Padova. P.M. acknowledge the Chistera EU project QUASAR. A.C. was supported by Project No. FIS2011-29400 (MINECO, Spain).

\section{APPENDIX: OPTIMALITY OF DETECTION STRATEGY FOR MAXIMALLY ENTANGLED STATES}

In this section we will demonstrate which is the optimal way of rewriting the Bell inequalities analyzed in the main text in case of maximally entangled states. We start by giving the general framework to solve the optimization.

Let us consider a general bipartite Bell inequality involving $m_{A}$ and $m_{B}$ observables $A_{j}$ and $B_{k}$ on the Alice and Bob side. The observables have $d_{A}$ and $d_{B}$ outcomes respectively, $\mu=1,2, \ldots, d_{A}$ and $v=1,2, \ldots, d_{B}$. Any Bell inequality can be written as (2)

$$
\langle\mathcal{S}\rangle_{\rho} \leqslant S_{\mathrm{LHV}}
$$


with

$$
\begin{aligned}
\mathcal{S}= & \sum_{j=1}^{m_{A}} \sum_{k=1}^{m_{A}} \sum_{\mu=1}^{d_{A}-1} \sum_{\nu=1}^{d_{A}-1} c_{j k}^{\mu v} p\left(a_{j}^{\mu} b_{k}^{\mu}\right) \\
& +\sum_{j=1}^{m_{A}} \sum_{\mu=1}^{d_{A}-1} \alpha_{j}^{\mu} p\left(a_{j}^{\mu}\right)+\sum_{k=1}^{m_{B}} \sum_{\nu=1}^{d_{B}-1} \beta_{k}^{v} p\left(b_{k}^{v}\right) .
\end{aligned}
$$

In the previous expression $p\left(a_{j}^{\mu} b_{j}^{\nu}\right)=p\left(A_{j}=\mu, B_{k}=v\right)$ are the joint probabilities of detecting the $\mu$ and $v$ eigenvectors $\left|a_{j}^{\mu}\right\rangle$ and $\left|b_{k}^{\nu}\right\rangle$ of the observables $A_{j}$ and $B_{k}$. Note that only the first $d_{A}-1$ and $d_{B}-1$ outcomes are involved in the inequality.

When inefficiencies are present it is necessary to give a strategy for the nondetection events. Let us suppose that the strategy on Alice's side is the following. If Alice is measuring the observable $A_{j}$ and the particle is not detected, she assigned, with probability $\mathcal{A}_{j}^{(\mu)}$, the outcome $\mu$. Clearly, $\sum_{\mu=1}^{d_{A}} \mathcal{A}_{j}^{(\mu)}=1$. The same happens at Bob's side, with probabilities $\mathcal{B}_{k}^{(\nu)}$. If we consider Alice and Bob inefficiencies as $\eta_{A}$ and $\eta_{B}$, the Bell inequality is violated if

$$
\begin{aligned}
& \eta_{A} \eta_{B}\langle\mathcal{S}\rangle_{\rho}+\left(1-\eta_{A}\right) \eta_{B} T_{A}+\eta_{A}\left(1-\eta_{B}\right) T_{B} \\
& \quad+\left(1-\eta_{A}\right)\left(1-\eta_{B}\right) X_{A B}>S_{\mathrm{LHV}}
\end{aligned}
$$

with

$$
\begin{aligned}
T_{A} & =\sum_{j, k, \mu, v} c_{j k}^{\mu \nu} \mathcal{A}_{j}^{(\mu)} p_{\rho}\left(b_{k}^{v}\right)+\sum_{j, \mu} \alpha_{j}^{\mu} \mathcal{A}_{j}^{(\mu)}+\sum_{k, v} \beta_{k}^{\nu} p_{\rho}\left(b_{k}^{v}\right), \\
T_{B} & =\sum_{j, k, \mu, v} c_{j k}^{\mu \nu} p_{\rho}\left(a_{j}^{\mu}\right) \mathcal{B}_{k}^{(v)}+\sum_{j, \mu} \alpha_{j}^{\mu} p_{\rho}\left(a_{j}^{\mu}\right)+\sum_{k, v} \beta_{k}^{\nu} \mathcal{B}_{k}^{(\nu)}, \\
X_{A B} & =\sum_{j, k, \mu, v} c_{j k}^{\mu \nu} \mathcal{A}_{j}^{(\mu)} \mathcal{B}_{k}^{(v)}+\sum_{j, \mu} \alpha_{j}^{\mu} \mathcal{A}_{j}^{(\mu)}+\sum_{k, v} \beta_{k}^{v} \mathcal{B}_{k}^{(\nu)} .
\end{aligned}
$$

The sum is taken over $i=1, \ldots, m_{A}$ and $j=1, \ldots, m_{B}$ while $\mu=1, \ldots, d_{A}-1$ and $v=1, \ldots, d_{B}-1$ : also in the previous expression the outcomes $d_{A}$ and $d_{B}$ of each observable are not present.

We start with the chained Bell inequalities, and then analyze the $I_{3322}$ and $I_{3}$ inequalities.

\section{Chained Bell inequalities}

For the chained Bell inequalities of Sec. III, we have dichotomic observables. Then, in the case of nondetection on the observable $A_{j}$, Alice chooses to output the +1 outcome with probability $\mathcal{A}_{j}$ and the -1 outcome with probability $1-\mathcal{A}_{j}$. The same happens to Bob. Remembering that, for MES, $p_{\rho}\left(a_{j}\right)=p_{\rho}\left(b_{k}\right)=\frac{1}{2}$ we have

$$
\begin{aligned}
T_{A}= & T_{B}=\frac{M-1}{2} \\
X_{A B}= & \mathcal{A}_{M} \mathcal{B}_{M}+\sum_{k=2}^{M}\left(\mathcal{A}_{k} \mathcal{B}_{k-1}+\mathcal{A}_{k-1} \mathcal{B}_{k}\right) \\
& -\mathcal{A}_{1} \mathcal{B}_{1}-\sum_{k=2}^{M}\left(\mathcal{A}_{k}+\mathcal{B}_{k}\right)
\end{aligned}
$$

Since $X_{A B}$ corresponds to the chained Bell inequality applied to the classical probabilities $\mathcal{A}_{k}$ and $\mathcal{B}_{k}$, we have $X_{A B} \leqslant 0$. In order to maximize the Bell parameter it is necessary to choose the $\mathcal{A}_{k}$ 's and $\mathcal{B}_{k}$ 's that maximize $X_{A B}$. The trivial choice $\mathcal{A}_{k}=$ $\mathcal{B}_{k}=0, \forall k$ satisfies this requirement. It is worth noticing that the choice $\mathcal{A}_{k}=\mathcal{B}_{k}=0, \forall k$, corresponds precisely to consider all nondetections as -1 outputs for the inequality written as (4).

\section{2. $I_{3322}$ inequality}

Let us consider the $I_{3322}$ inequality written in its original form $\quad I_{3322}=p\left(a_{1} b_{1}\right)+p\left(a_{1} b_{2}\right)+p\left(a_{1} b_{3}\right)+p\left(a_{2} b_{1}\right)+$ $p\left(a_{2} b_{2}\right)+p\left(a_{3} b_{1}\right)-p\left(a_{2} b_{3}\right)-p\left(a_{3} b_{2}\right)-2 p\left(a_{1}\right)-p\left(a_{2}\right)-$ $p\left(b_{1}\right)$. In the case of inefficiencies with nonmaximally entangled states we have

$$
\begin{aligned}
T_{A}= & -\frac{1}{2}\left(\mathcal{A}_{1}+\mathcal{A}_{2}+1\right), \quad T_{B}=\frac{1}{2}\left(\mathcal{B}_{1}+\mathcal{B}_{2}-3\right), \\
X_{A B}= & \mathcal{A}_{3}\left(\mathcal{B}_{1}-\mathcal{B}_{2}\right)+\mathcal{B}_{3}\left(\mathcal{A}_{1}-\mathcal{A}_{2}\right)+\mathcal{A}_{1} \mathcal{B}_{1}+\mathcal{A}_{1} \mathcal{B}_{2} \\
& +\mathcal{A}_{2} \mathcal{B}_{1}+\mathcal{A}_{2} \mathcal{B}_{2}-2 \mathcal{A}_{1}-\mathcal{A}_{2}-\mathcal{B}_{1} .
\end{aligned}
$$

Since the maximal value of $\left\langle I_{3322}\right\rangle$ with maximally entangled state is $1 / 4$, the Bell parameter in the case of detection inefficiencies $\eta_{A}=\eta_{B}=\eta$ becomes

$\frac{1}{4} \eta^{2}+\frac{1}{2} \eta(1-\eta)\left(\mathcal{B}_{1}+\mathcal{B}_{2}-\mathcal{A}_{1}-\mathcal{A}_{2}-4\right)+(1-\eta)^{2} X_{A B}$.

The choice that minimizes the critical efficiency is given by $\mathcal{B}_{1}=\mathcal{B}_{2}=1$ and $\mathcal{B}_{3}=\mathcal{A}_{1}=\mathcal{A}_{2}=\mathcal{A}_{3}=0$, giving $X_{A B}=$ $-1, T_{A}=T_{B}=-\frac{1}{2}$, and

$$
\frac{1}{4} \eta^{2}-\eta(1-\eta)-(1-\eta)^{2}>0,
$$

solved by

$$
\eta>2(\sqrt{2}-1) \simeq 0.828 .
$$

The choice of the $\mathcal{A}$ 's and $\mathcal{B}$ 's corresponds to choosing for the nondetection events the outcome -1 for the inequality written as $I_{3322}^{(2)}$.

\section{Two-qutrit $I_{3}$ inequality}

For this two-qutrit inequality Alice has three outcomes for each observable $A_{j}$. In the case of nondetection she assigns with probability $\mathcal{A}_{j}^{(1)}$ the outcome 1 , with probability $\mathcal{A}_{j}^{(2)}$ the outcome 2 , and with probability $1-\mathcal{A}_{j}^{(1)}-\mathcal{A}_{j}^{(2)}$ the outcome 3 . The same applies to Bob. For maximally entangled states $p\left(a_{j}\right)=p\left(b_{j}\right)=p\left(\bar{a}_{j}\right)=p\left(\bar{b}_{j}\right)=\frac{1}{3}$, and we have

$$
\begin{aligned}
T_{A}= & T_{B}=-\frac{2}{3}, \\
X_{A B}= & \mathcal{A}_{1}^{(1)} \mathcal{B}_{1}^{(1)}+\mathcal{A}_{1}^{(1)} \mathcal{B}_{2}^{(1)}+\mathcal{A}_{2}^{(1)} \mathcal{B}_{1}^{(1)}-\mathcal{A}_{2}^{(1)} \mathcal{B}_{2}^{(1)} \\
& +\mathcal{A}_{1}^{(2)} \mathcal{B}_{1}^{(2)}+\mathcal{A}_{1}^{(2)} \mathcal{B}_{2}^{(2)}+\mathcal{A}_{2}^{(2)} \mathcal{B}_{1}^{(2)}-\mathcal{A}_{2}^{(2)} \mathcal{B}_{2}^{(2)} \\
& +\mathcal{A}_{1}^{(1)} \mathcal{B}_{1}^{(2)}+\mathcal{A}_{1}^{(2)} \mathcal{B}_{2}^{(1)}+\mathcal{A}_{2}^{(2)} \mathcal{B}_{1}^{(1)}-\mathcal{A}_{2}^{(2)} \mathcal{B}_{2}^{(1)} \\
& -\mathcal{A}_{1}^{(1)}-\mathcal{A}_{1}^{(2)}-\mathcal{B}_{1}^{(1)}-\mathcal{B}_{1}^{(2)} .
\end{aligned}
$$


The optimal choice of $\mathcal{A}_{k}^{(\mu)}$,s and $\mathcal{B}_{k}^{(\mu)}$, s is the one that maximizes $X_{A B}$. This term is clearly upper bounded by 0 (it corresponds to the Bell inequality). Then the choice
$\mathcal{A}_{j}^{(\mu)}=\mathcal{B}_{k}^{(\nu)}=0$ saturates the bound. This choice corresponds to choosing for the nondetection events the outcome 2 for the inequality written as (25).
[1] J. S. Bell, Physics 1, 195 (1964).

[2] N. Gisin, Phys. Lett. A 154, 201 (1991).

[3] R. F. Werner and M. M. Wolf, Phys. Rev. A 61, 062102 (2000).

[4] A. Acín, R. Gill, and N. Gisin, Phys. Rev. Lett. 95, 210402 (2005).

[5] A. Acín, T. Durt, N. Gisin, and J. I. Latorre, Phys. Rev. A 65, 052325 (2002).

[6] D. Collins, N. Gisin, N. Linden, S. Massar, and S. Popescu, Phys. Rev. Lett. 88, 040404 (2002).

[7] M. Junge and C. Palazuelos, Commun. Math. Phys. 306, 695 (2011).

[8] T. Vidick and S. Wehner, Phys. Rev. A 83, 052310 (2011).

[9] Y.-C. Liang, T. Vértesi, and N. Brunner, Phys. Rev. A 83, 022108 (2011).

[10] W. K. Wootters, Phys. Rev. Lett. 80, 2245 (1998).

[11] P. Rungta, V. Bužek, C. M. Caves, M. Hillery, and G. J. Milburn, Phys. Rev. A 64, 042315 (2001).

[12] D. Collins and N. Gisin, J. Phys. A: Math. Gen. 37, 1775 (2004).

[13] P. M. Pearle, Phys. Rev. D 2, 1418 (1970).

[14] N. Brunner and N. Gisin, Phys. Lett. A 372, 3162 (2008).

[15] S. Massar, S. Pironio, J. Roland, and B. Gisin, Phys. Rev. A 66, $052112(2002)$

[16] J.-Å. Larsson, Phys. Rev. A 57, 3304 (1998).

[17] J. F. Clauser, M. A. Horne, A. Shimony, and R. A. Holt, Phys. Rev. Lett. 23, 880 (1969).

[18] P. H. Eberhard, Phys. Rev. A 47, R747 (1993).

[19] A. Cabello and J.-A. Larsson, Phys. Rev. Lett. 98, 220402 (2007).

[20] N. Brunner, N. Gisin, V. Scarani, and C. Simon, Phys. Rev. Lett. 98, 220403 (2007).

[21] J.-Å. Larsson and J. Semitecolos, Phys. Rev. A 63, 022117 (2001).
[22] T. Vértesi, S. Pironio, and N. Brunner, Phys. Rev. Lett. 104, 060401 (2010).

[23] H. M. Wiseman, S. J. Jones, and A. C. Doherty, Phys. Rev. Lett. 98, 140402 (2007).

[24] G. Vallone, Phys. Rev. A 87, 020101 (2013).

[25] S. L. Braunstein and C. M. Caves, in Bell's Theorem, Quantum Theory, and Conceptions of the Universe, edited by M. Kafatos (Kluwer, Dordrecht, 1989), p. 27.

[26] S. L. Braunstein and C. M. Caves, Ann. Phys. (NY) 202, 22 (1990).

[27] J. F. Clauser and M. A. Horne, Phys. Rev. D 10, 526 (1974).

[28] S. Aerts, P. Kwiat, J.-Å. Larsson, and M. Żukowski, Phys. Rev. Lett. 83, 2872 (1999).

[29] A. Peres, Fortschr. Phys. 48, 531 (2000).

[30] J. Barrett, L. Hardy, and A. Kent, Phys. Rev. Lett. 95, 010503 (2005).

[31] J. Barrett, A. Kent, and S. Pironio, Phys. Rev. Lett. 97, 170409 (2006).

[32] G. Vallone, I. Gianani, E. B. Inostroza, C. Saavedra, G. Lima, A. Cabello, and P. Mataloni, Phys. Rev. A 83, 042105 (2011).

[33] D. Boschi, S. Branca, F. De Martini, and L. Hardy, Phys. Rev. Lett. 79, 2755 (1997).

[34] M. Barbieri, F. De Martini, G. Di Nepi, and P. Mataloni, Phys. Lett. A 334, 23 (2005).

[35] C. Dhara, G. Prettico, and A. Acín, Phys. Rev. A 88, 052116 (2013).

[36] A. Cabello, J.-^̊. Larsson, and D. Rodríguez, Phys. Rev. A 79, 062109 (2009).

[37] S. Wehner, Phys. Rev. A 73, 022110 (2006).

[38] M. Froissart, Nuovo Cimento B 64, 241 (1981).

[39] C. Śliwa, Phys. Lett. A 317, 165 (2003). 